Short communication

\title{
Objective measures of unobstructed walking and obstacle avoidance in Parkinson's disease subtypes
}

\author{
Diego Orcioli-Silva $^{\mathrm{a}, *}$, Rodrigo Vitório ${ }^{\mathrm{a}}$, Ellen Lirani-Silva ${ }^{\mathrm{a}}$, Paulo Cezar Rocha Santos ${ }^{\mathrm{a}, \mathrm{b}}$, \\ Victor Spiandor Beretta ${ }^{\mathrm{a}}$, Lilian Teresa Bucken Gobbi ${ }^{\mathrm{a}}$ \\ a São Paulo State University (UNESP), Institute of Biosciences, Posture and Gait Studies Laboratory (LEPLO), Rio Claro, Brazil \\ ${ }^{\mathrm{b}}$ University of Groningen, University Medical Center Groningen, Center for Human Movement Sciences, Groningen, The Netherlands
}

\section{A R T I C L E I N F O}

\section{Keywords:}

Gait

Obstacle avoidance

Parkinson's disease

PIGD

Tremor dominant

\begin{abstract}
A B S T R A C T
Background: Objective measures of gait in Parkinson's disease (PD) patients according to motor subtypes are not yet fully understood. Although recent advances have been made for unobstructed walking, further work is required on locomotor tasks challenging postural stability, such as obstacle avoidance.

Research question: This study aimed to investigate the influence of PD motor subtypes on objective measures of locomotion during unobstructed walking and obstacle avoidance.

Methods: Thirty-five PD patients classified as postural instability and gait disorder (PIGD) and 30 as tremor dominant (TD), as well as 45 healthy controls (CG) walked along an 8-m pathway under two conditions: (a) unobstructed walking and (b) obstacle avoidance. Outcome measures included spatiotemporal parameters recorded by an optoelectronic tridimensional system.

Results: During unobstructed walking, the PIGD group exhibited shorter stride length, slower velocity, and longer double support phase compared to the TD and CG groups. The TD group also presented slower stride velocity compared to the CG. The PIGD and TD groups presented shorter stride duration than the CG. Regarding obstacle avoidance, the PIGD group exhibited shorter distances for leading foot placement before obstacle, trailing foot placement after obstacle and trailing crossing step length compared to the TD and CG groups. The PIGD group exhibited wider leading crossing step width, lower trailing toe clearance, and slower leading and trailing velocity during obstacle avoidance compared to the CG.

Significance: PIGD subtype patients showed worse modifications in objective measures of unobstructed walking and obstacle avoidance. The observed modifications may contribute to increased fall occurrence in PIGD patients.
\end{abstract}

\section{Introduction}

Parkinson's disease (PD) is a heterogeneous neurodegenerative disorder with variable clinical characteristics [1]. Typically, patients are classified into different motor subtypes, including tremor dominant (TD) and postural instability and gait disorder (PIGD). TD is characterized by the predominant presence of tremors and PIGD is associated with more severe gait impairments, postural instability, bradykinesia, rigidity, and falling [1-4]. The influence of PD motor subtypes on objective measures of locomotion has not yet been fully understood. Although recent advances have been made for unobstructed walking (with conflicting findings) and dual tasking [3,4], further work is required on locomotor tasks challenging postural stability (e.g., obstacle avoidance). To date, previous studies have shown that obstacle avoidance is impaired in patients with PD [5-7], but with no clear information regarding PD subtypes. The development of this knowledge may help to better understand the differences between PIGD and TD subtypes and to tailor therapy. Thus, this study aimed to investigate the influence of PD motor subtypes on objective measures of locomotion during unobstructed walking and obstacle avoidance. We hypothesized that objective measures of unobstructed walking and obstacle avoidance would be worse among patients in the PIGD subtype.

\section{Methods}

\subsection{Participants}

Seventy-six patients with PD and 45 healthy controls (CG) were

\footnotetext{
* Corresponding author at: São Paulo State University (UNESP), Bioscience Institute, Campus Rio Claro, Posture and Gait Studies Laboratory (LEPLO), Avenida 24-A, 1515, Bela Vista, 13506-900, Rio Claro, SP, Brazil.

E-mail address: diegoos@rc.unesp.br (D. Orcioli-Silva).
} 
recruited. Patients with PD were classified into PIGD $(\mathrm{n}=35)$, TD $(\mathrm{n}=30)$, or indeterminate $(\mathrm{n}=11)$ according to the procedures proposed by Jankovic et al. [1]. Patients with PD were included if they were: (a) diagnosed with idiopathic PD (b) in Hoehn and Yahr (HY) stages I-III; and (c) taking PD medication. Participants were excluded if they had a diagnosis of dementia or co-morbidities likely to affect gait. The Unified Parkinson's Disease Rating Scale (UPDRS) [8] and the HY [9] were used to determine the severity and stage of the disease in the patients with PD. Global cognition of all participants was assessed using the Mini Mental State Examination (MMSE) [10]. Patients within indeterminate subtypes were excluded from further analysis. PD groups were tested during the "on state" of PD medication. All participants selfreported the number of falls during the previous 12 months. This study was approved by the local ethics committee. All subjects gave written informed consent.

\subsection{Procedures}

Gait was assessed under two conditions (at self-selected speed): unobstructed walking and obstacle avoidance. The obstacle was set up at half knee height $\times 60 \mathrm{~cm}$ width $\times 3 \mathrm{~cm}$ depth [6]. The obstacle was positioned in the middle of a pathway ( $8 \mathrm{~m}$ long). Three unobstructed walking trials and six obstacle avoidance trials, comprising equal numbers of left and right crossing steps (fully randomized) were analyzed.

Gait parameters were recorded using an optoelectronic tridimensional system (OPTOTRAK Certus, $100 \mathrm{~Hz}$ ), positioned in the right sagittal plane. Four active markers were attached to the 5th metatarsal and lateral face of the calcaneus of the right foot and 1st metatarsal and medial face of the calcaneus of the left foot. One marker was fixed at the top edge of the obstacle. Marker trajectories were filtered with a fifthorder Butterworth low-pass filter, with a cutoff frequency of $6 \mathrm{~Hz}$. The spatiotemporal parameters of unobstructed walking (mid-pathway right stride) and obstacle avoidance (mean of six trials) (Fig. 1) were calculated using an algorithm created in Matlab 7.0 (The Maths Works Inc.). Further details about data processing and outcome variables can be found in earlier studies by our group [6,7].

\subsection{Statistical analysis}

Demographic data were analyzed by ANOVA, Pearson's Chi-square test (sex), and independent-samples $t$-test (PD-related). Spatiotemporal parameters were analyzed by ANOVA; the mean value of three (unobstructed walking) or six trials (obstacle avoidance) was considered in the analysis $(p \leq 0.05)$. The Bonferroni post hoc test was used to localize the differences among groups ( $P$-value was adjusted).

\section{Results}

The group characteristics are shown in Table 1. As expected, PIGD patients reported a greater number of falls than TD patients and the CG. In addition, PIGD patients demonstrated worse scores in the UPDRS I, UPDRS II, and UPDRS total compared to TD patients.

For unobstructed walking (Table 2), the PIGD group presented shorter stride length $(\mathrm{p}=0.001, \mathrm{p}<0.001)$, slower velocity $(\mathrm{p}=0.034, \mathrm{p}<0.001)$, and longer double support phase $(\mathrm{p}=0.015$, $\mathrm{p}=0.002$ ) compared to the TD and CG groups. The TD group also demonstrated slower stride velocity compared to the CG ( $\mathrm{p}=0.010)$. In addition, the PIGD and TD groups presented shorter stride duration than the CG ( $\mathrm{p}=0.013, \mathrm{p}=0.034)$.

During obstacle avoidance (Table 2), the PIGD group exhibited shorter distances for leading foot placement before obstacle ( $\mathrm{p}=0.05$, $\mathrm{p}=0.015)$, trailing foot placement after obstacle $(\mathrm{p}=0.029$, $\mathrm{p}<0.001)$, and trailing crossing step length $(\mathrm{p}=0.014, \mathrm{p}<0.001)$ compared to the TD and CG groups. The PIGD group exhibited wider leading crossing step width $(\mathrm{p}=0.016)$, lower trailing toe clearance $(\mathrm{p}=0.031)$, and slower leading $(\mathrm{p}<0.001)$ and trailing $(\mathrm{p}<0.001)$ velocity during obstacle avoidance $(\mathrm{p}=0.001)$ compared to the CG.

\section{Discussion}

The current study compared PIGD and TD subtypes of PD during unobstructed walking and obstacle avoidance. Our findings confirmed our hypotheses, supporting that the objective measures of both walking conditions are worse in PIGD patients. Hypometria and bradykinesia are exacerbated in PIGD patients in both unobstructed walking and obstacle avoidance.

Regarding unobstructed walking, it is important to highlight that
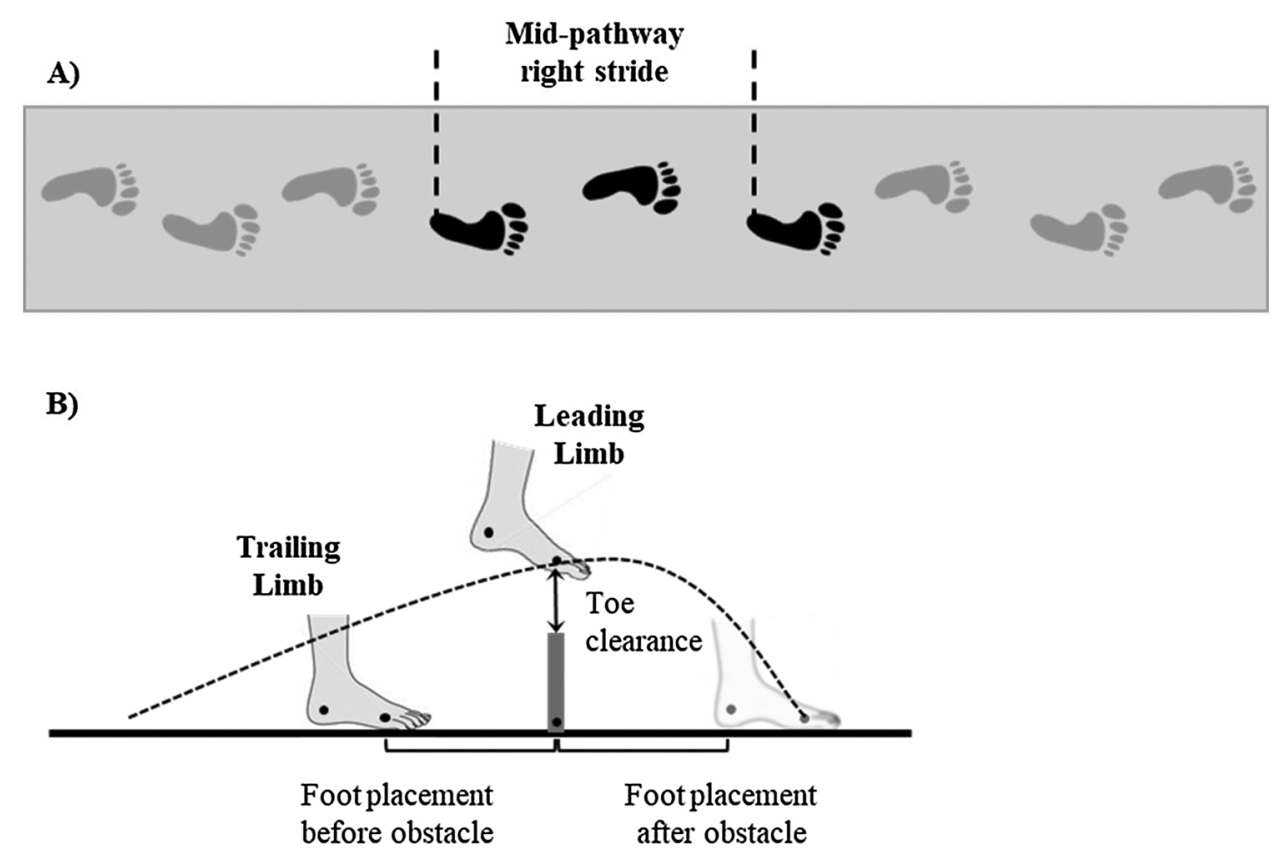

Fig. 1. Representation of the spatial dependent variables during unobstructed walking (A) and obstacle avoidance (B). 
Table 1

Groups' characteristics.

\begin{tabular}{|c|c|c|c|c|}
\hline Demographic measures & $\mathrm{TD}$ & PIGD & CG & p-value \\
\hline Sex (male/female) & $20 / 10$ & $17 / 18$ & $21 / 24$ & 0.197 \\
\hline Age (years) & $69.86 \pm 6.97$ & $67.66 \pm 9.38$ & $66.51 \pm 8.64$ & 0.247 \\
\hline Body height $(\mathrm{cm})$ & $163.50 \pm 8.15$ & $158.90 \pm 9.11$ & $160.98 \pm 7.52$ & 0.084 \\
\hline Body mass (Kg) & $73.43 \pm 10.61$ & $67.50 \pm 14.80$ & $69.90 \pm 13.02$ & 0.191 \\
\hline MMSE (0-30) & $28.13 \pm 1.76$ & $27.66 \pm 2.40$ & $28.07 \pm 2.04$ & 0.591 \\
\hline Number of falls & $0.33 \pm 0.61$ & $4.57 \pm 7.51$ & $0.20 \pm 0.51$ & $<.001^{\mathrm{a}, \mathrm{b}}$ \\
\hline Disease duration (years) & $6.25 \pm 5.24$ & $6.17 \pm 4.45$ & NA & 0.948 \\
\hline UPDRS - I (0-16) & $2.80 \pm 1.47$ & $4.00 \pm 1.93$ & NA & $.007^{\mathrm{a}}$ \\
\hline UPDRS - II (0-52) & $9.47 \pm 4.49$ & $13.86 \pm 6.47$ & NA & $.003^{\mathrm{a}}$ \\
\hline UPDRS - III (0-108) & $23.03 \pm 8.04$ & $25.03 \pm 11.08$ & NA & 0.416 \\
\hline UPDRS - total (0-176) & $35.30 \pm 11.64$ & $42.89 \pm 17.20$ & NA & $.045^{\mathrm{a}}$ \\
\hline
\end{tabular}

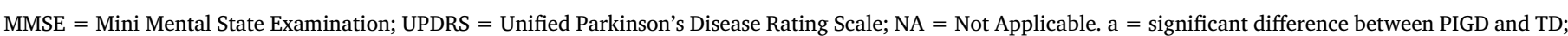
$\mathrm{b}=$ significant difference between PIGD and CG.

Table 2

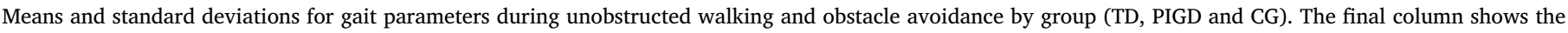
group main effects.

\begin{tabular}{|c|c|c|c|c|}
\hline Gait parameters & TD & PIGD & CG & ANOVA \\
\hline \multicolumn{5}{|l|}{ Unobstructed walking } \\
\hline Stride length $(\mathrm{cm})$ & $119.20 \pm 14.83$ & $102.77 \pm 25.18$ & $127.65 \pm 13.36$ & $\mathrm{~F}_{2,107}=18.405, \mathrm{p}<0.001, \eta^{2}=0.256^{\mathrm{a}, \mathrm{b}}$ \\
\hline Stride duration (s) & $1.10 \pm 0.13$ & $1.11 \pm 0.19$ & $1.02 \pm 0.09$ & $F_{2,107}=5.347, p=0.006, \eta^{2}=0.091 b, c$ \\
\hline Stride velocity $(\mathrm{cm} / \mathrm{s})$ & $110.31 \pm 21.75$ & $95.52 \pm 28.74$ & $126.57 \pm 18.53$ & $\mathrm{~F}_{2,107}=17.991, \mathrm{p}<0.001, \eta^{2}=0.252^{\mathrm{a}, \mathrm{b}, \mathrm{c}}$ \\
\hline Double support (\%) & $18.08 \pm 7.38$ & $22.67 \pm 8.34$ & $17.61 \pm 3.28$ & $F_{2,107}=6.909, p=0.002, \eta^{2}=0.114^{a, b}$ \\
\hline \multicolumn{5}{|l|}{ Obstacle avoidance - Leading limb } \\
\hline Crossing step length $(\mathrm{cm})$ & $64.17 \pm 7.28$ & $60.96 \pm 9.38$ & $64.92 \pm 7.26$ & $\mathrm{~F}_{2,107}=2.567, \mathrm{p}=0.081, \eta^{2}=0.046$ \\
\hline Crossing step width $(\mathrm{cm})$ & $10.85 \pm 3.99$ & $12.01 \pm 4.54$ & $9.58 \pm 2.89$ & $\mathrm{~F}_{2,107}=4.105, \mathrm{p}=0.019, \eta^{2}=0.071^{b}$ \\
\hline Foot placement before obstacle $(\mathrm{cm})$ & $80.50 \pm 14.13$ & $70.96 \pm 20.31$ & $81.23 \pm 12.81$ & $F_{2,107}=4.716, p=0.011, \eta^{2}=0.081$ \\
\hline Foot placement after obstacle $(\mathrm{cm})$ & $21.84 \pm 5.43$ & $20.10 \pm 4.96$ & $22.78 \pm 4.81$ & $\mathrm{~F}_{2,107}=2.812, \mathrm{p}=0.065, \eta^{2}=0.050$ \\
\hline Toe clearance $(\mathrm{cm})$ & $16.82 \pm 3.83$ & $16.15 \pm 4.81$ & $17.93 \pm 3.47$ & $\mathrm{~F}_{2,107}=2.005, \mathrm{p}=0.140, \eta^{2}=0.036$ \\
\hline Horizontal velocity during obstacle crossing $(\mathrm{cm} / \mathrm{s})$ & $156.98 \pm 37.53$ & $140.19 \pm 46.16$ & $176.05 \pm 32.77$ & $\mathrm{~F}_{2,107}=8.522, \mathrm{p}<0.001, \eta^{2}=0.137^{\mathrm{b}}$ \\
\hline \multicolumn{5}{|l|}{ Obstacle avoidance - Trailing limb } \\
\hline Crossing step length $(\mathrm{cm})$ & $52.43 \pm 10.62$ & $43.78 \pm 13.27$ & $55.68 \pm 11.92$ & $\mathrm{~F}_{2,107}=9.906, \mathrm{p}<0.001, \mathrm{\eta}^{2}=0.156^{\mathrm{a}, \mathrm{b}}$ \\
\hline Crossing step width $(\mathrm{cm})$ & $13.78 \pm 5.34$ & $14.71 \pm 5.57$ & $13.58 \pm 5.57$ & $\mathrm{~F}_{2,107}=0.451, \mathrm{p}=0.638, \eta^{2}=0.008$ \\
\hline Foot placement before obstacle $(\mathrm{cm})$ & $26.11 \pm 4.99$ & $25.48 \pm 5.58$ & $25.10 \pm 4.38$ & $\mathrm{~F}_{2,107}=0.375, \mathrm{p}=0.688, \eta^{2}=0.007$ \\
\hline Foot placement after obstacle $(\mathrm{cm})$ & $74.26 \pm 14.48$ & $63.88 \pm 17.25$ & $78.45 \pm 15.57$ & $\mathrm{~F}_{2,107}=8.531, \mathrm{p}<0.001, \eta^{2}=0.138^{\mathrm{a}, \mathrm{b}}$ \\
\hline Toe clearance $(\mathrm{cm})$ & $24.08 \pm 5.08$ & $23.02 \pm 6.33$ & $26.55 \pm 6.26$ & $F_{2,107}=3.674, p=0.029, \eta^{2}=0.064^{b}$ \\
\hline Horizontal velocity during obstacle crossing $(\mathrm{cm} / \mathrm{s})$ & $178.65 \pm 37.66$ & $166.18 \pm 50.04$ & $201.36 \pm 39.68$ & $F_{2,107}=6.988, p=0.001, \eta^{2}=0.111$ \\
\hline
\end{tabular}

$\mathrm{a}=$ significant difference between PIGD and TD; $\mathrm{b}=$ significant difference between PIGD and CG; $\mathrm{c}=$ significant difference between TD and CG.

conflicting findings have been reported [3,4]. While Herman's study found no differences between PIGD and TD subtypes, Vervoort's study observed slower gait speed and shorter step length in the PIGD patients. Our findings corroborate with Vervoort's findings and suggest that objective gait measures are consistent with clinical aspects of motor subtypes in PD.

Safe locomotion over uneven terrains requires the ability to adequately avoid obstacles while walking. Tripping over obstacles has been identified as a major cause of falls in PD $[11,12]$ and reductions in foot to obstacle distances, which were observed in PIGD patients only, have been shown to lead to greater number of obstacle contacts during crossing [13]. As such, the observed reductions in foot to obstacle distances in PIGD patients may lead them to a greater risk of tripping and, therefore, may be associated with the greater number of falls reported by these patients (Table 1). Additionally, PIGD patients showed behavioral changes commonly associated with a 'cautious strategy' [14]: increased step width guarantees the center of mass projection within the boundaries of the base of support. Another aspect that requires clarification is the fact that previous studies, which included both TD and PIGD subtypes in the same group, have argued that obstacle avoidance is impaired in patients with PD (in general) [5-7]. Current findings, however, highlight the importance of treating PD subtype as a factor in the analysis; PIDG patients may have driven the observed differences in previous studies.

There is a warm debate in the literature about the best classification method to deal with PD heterogeneity. New methods have been argued to overcome existing limitations of the traditional method $[3,15]$. Although this study does not directly compare different methods, we used the traditional method instead, current findings suggest that clinical assessments of the disturbances seen in the PIGD subtype (traditional classification) are consistent with objective measures of unobstructed walking and obstacle avoidance.

In conclusion, PIGD patients showed worse modifications in objective measures of unobstructed walking and obstacle avoidance. The observed modifications may contribute to increased fall occurrence in PIGD patients.

\section{Conflict of interest}

The authors have no conflicts of interest to disclose.

\section{Acknowledgement}

The authors thank São Paulo Research Foundation (FAPESP) (grant numbers: \#2010/07040-0; \#2013/27032-0; \#2014/22308-0) for financial support.

\section{References}

[1] J. Jankovic, M. McDermott, J. Carter, S. Gauthier, C. Goetz, L. Golbe, et al., Variable 
expression of Parkinson's disease: a base-line analysis of the DATATOP cohort. The Parkinson Study Group, Neurology 40 (1990) 1529-1534.

[2] B. Galna, S. Lord, D.J. Burn, L. Rochester, Progression of gait dysfunction in incident Parkinson's disease: impact of medication and phenotype, Mov. Disord. 30 (2015) 359-367.

[3] T. Herman, A. Weiss, M. Brozgol, N. Giladi, J.M. Hausdorff, Gait and balance in Parkinson's disease subtypes: objective measures and classification considerations, J. Neurol. 261 (2014) 2401-2410.

[4] G. Vervoort, A. Bengevoord, E. Nackaerts, E. Heremans, W. Vandenberghe, A. Nieuwboer, Distal motor deficit contributions to postural instability and gait disorder in Parkinson's disease, Behav. Brain Res. 287 (2015) 1-7.

[5] D. Orcioli-Silva, F.A. Barbieri, L. Simieli, R. Vitorio, P. Santos, V.S. Beretta, et al. Walking behavior over multiple obstacles in people with Parkinson's disease, Gait Posture 58 (2017) 510-515.

[6] R. Vitorio, E. Lirani-Silva, A.M. Baptista, F.A. Barbieri, P.C. dos Santos, C. TeixeiraArroyo, et al., Disease severity affects obstacle crossing in people with Parkinson's disease, Gait Posture 40 (2014) 266-269.

[7] R. Vitorio, F. Pieruccini-Faria, F. Stella, S. Gobbi, L.T. Gobbi, Effects of obstacle height on obstacle crossing in mild Parkinson's disease, Gait Posture 31 (2010) $143-146$.

[8] R.J. Uitti, Y. Baba, Z.K. Wszolek, D.J. Putzke, Defining the Parkinson's disease phenotype: initial symptoms and baseline characteristics in a clinical cohort,
Parkinsonism Relat. Disord. 11 (2005) 139-145

[9] M.M. Hoehn, M.D. Yahr, Parkinsonism: onset, progression and mortality, Neurology 17 (1967) 427-442.

[10] S.M. Brucki, R. Nitrini, P. Caramelli, P.H. Bertolucci, I.H. Okamoto, [Suggestions for utilization of the mini-mental state examination in Brazil], Arq. Neuropsiquiatr. 61 (2003) 777-781.

[11] A. Ashburn, E. Stack, C. Ballinger, L. Fazakarley, C. Fitton, The circumstances of falls among people with Parkinson's disease and the use of Falls Diaries to facilitate reporting, Disabil. Rehabil. 30 (2008) 1205-1212.

[12] T. Gazibara, D. Kisic Tepavcevic, M. Svetel, A. Tomic, I. Stankovic, V.S. Kostic, et al., Near-falls in people with Parkinson's disease: circumstances, contributing factors and association with falling, Clin. Neurol. Neurosurg. 161 (2017) 51-55.

[13] L.S. Chou, L.F. Draganich, Placing the trailing foot closer to an obstacle reduces flexion of the hip, knee, and ankle to increase the risk of tripping, J. Biomech. 31 (1998) 685-691.

[14] E.L. Stegemoller, T.A. Buckley, C. Pitsikoulis, E. Barthelemy, R. Roemmich, C.J. Hass, Postural instability and gait impairment during obstacle crossing in Parkinson's disease, Arch. Phys. Med. Rehabil. 93 (2012) 703-709.

[15] R. Erro, C. Vitale, M. Amboni, M. Picillo, M. Moccia, K. Longo, et al., The heterogeneity of early Parkinson's disease: a cluster analysis on newly diagnosed untreated patients, PLoS One 8 (2013) e70244. 